\title{
Calculation of the structure and absorption spectra of phthalocyanines in the gas-phase and in solution
}

\author{
P.N. Day, Zhiqiang Wang, R. Pachter* \\ Materials Directorate, Wright Laboratory, Wright-Patterson Air Force Base, OH 45433, USA \\ Received 10 March 1998; received in revised form 5 May 1998; accepted 5 May 1998
}

\begin{abstract}
$\mathrm{Ab}$ initio calculations have been carried out on both gas-phase and solvated phthalocyanines in order to determine equilibrium structures, vibrational spectra, and electronic spectra. Density functional theory (DFT) optimized geometries have the expected symmetries of $D_{2 \mathrm{~h}}$ for free-base phthalocyanine, $D_{4 \mathrm{~h}}$ for copper phthalocyanine, and $C_{4 \mathrm{v}}$ for tin phthalocyanine, whereas HartreeFock optimized geometries, which also include lead phthalocyanine, are slightly displaced from the expected symmetries. Both sets of optimized geometries agree reasonably well with the available experimental structures. Vibrational spectra, calculated by the Hartree-Fock method, are in partial agreement with measured spectra, whereas visible absorption spectra calculated using molecular geometries obtained at three different levels of theory (DFT, HF, and PM3) showed good agreement with the measured vapor-phase spectra in the Q-band, and using the DFT optimized geometry resulted in very good agreement with measurements in the B-band. The self-consistent reaction field method for including bulk solvent effects in HF calculations had a small, nearly negligible, effect on the molecular structure of SnPc, whereas the DFT/COSMO solvation model yielded the predictable result of increasing the Sn-N bond length by pulling the $\mathrm{Sn}$ atom further out of the molecular plane. (C) 1998 Elsevier Science B.V. All rights reserved.
\end{abstract}

Keywords: Phthalocyanines; Visible spectra; Vibrational spectra; Solvation effects

\section{Introduction}

The excited-state absorption properties of metallophthalocyanines [1] make them of interest for optical limiting applications [2-4]. In order to enhance optical limiting properties and tune the absorption spectra, a series of calculations on these derivatives was carried out. However, reasonably "good" geometries for spectra predictions have to be first obtained [5]. Ab initio Hartree-Fock calculations

\footnotetext{
* Corresponding author.
}

have been carried out on four phthalocyanine compounds: the metal-free phthalocyanine $\left(\mathrm{H}_{2} \mathrm{Pc}\right)$, copper phthalocyanine $(\mathrm{CuPc})$, tin phthalocyanine $(\mathrm{SnPc})$, and lead phthalocyanine $(\mathrm{PbPc})$. Density functional theory (DFT) calculations have been carried out on $\mathrm{H}_{2} \mathrm{Pc}$, $\mathrm{CuPc}$ and SnPc. Note that experimental structures are available for these molecules from X-ray diffraction or neutron diffraction studies. X-ray crystal structures are available for $\mathrm{CuPc}$ [6], SnPc [7], and for two structures of $\mathrm{PbPc}$, from both the monoclinic crystal [8] and the triclinic crystal [9]. A geometry for $\mathrm{H}_{2} \mathrm{Pc}$ is available from a neutron diffraction study [10]. 
$\mathrm{Ab}$ initio calculations on copper phthalocyanine have been previously carried out by Szczepaniak and Bragiel [11]. However, a relatively small basis set (3-21G) was used and no geometry optimization was carried out. Instead, the geometry from the X-ray diffraction crystal structure study [6] was employed (modified to an idealized $D_{4 \mathrm{~h}}$ symmetry). In this study, we report geometry optimizations at both the Hartree-Fock and DFT levels of theory to estimate equilibrium geometries of the isolated $\mathrm{H}_{2} \mathrm{Pc}, \mathrm{CuPc}$, $\mathrm{SnPc}$, and $\mathrm{PbPc}$ molecules and to evaluate the appropriate approach to be used for organometallic systems of interest to us. The geometries obtained by both theoretical methods agree well with experiment. The DFT method was more successful at obtaining the expected gas-phase symmetry. The structures obtained for the free-base phthalocyanine were used to evaluate the vibrational spectra via calculation and diagonalization of the Hessian matrix, and a fair agreement is indicated with the available experimental values.

Both calculated and experimental geometries were used to calculate electronic absorption spectra using configuration interaction (CI) within a semiempirical formalism, INDO/s. The electronic absorption spectra for phthalocyanines show a strong peak in the blue (300-400 nm) region, referred to as the B-band, and an even stronger Q-band in the red to near-infrared $(600-700 \mathrm{~nm})$. This differs from the spectra of porphyrins, where the Q-band tends to be much weaker than the B-band. The spectra for free-base phthalocyanine were calculated using three computationally optimized geometries (HF, DFT, and PM3) and one experimental geometry. For the freebase phthalocyanine, the Q-band is split into two strong peaks, and the calculations from all four geometries correctly predicted this result, whereas only the more symmetrical DFT structure was successful in predicting the single strong peak in the B-band for the gas-phase free-base phthalocyanine.

These structures can also be used as starting points for evaluating the influence of a solvent. The effects of solvation on these molecules have been investigated using continuum reaction field models. The use of such a model with DFT predicted a reasonable deviation from the gas-phase geometry for tin phthalocyanine, whereas no appreciable change in the geometry was found with the self-consistent reaction field (SCRF) included in the Hartree-Fock calculation.

\section{Methods}

The GAMESS [12] program was used to carry out the Hartree-Fock and semiempirical calculations. $\mathrm{DMOL}^{1}$ [13] was used for the DFT calculations. Restricted-spin Hartree-Fock (RHF) calculations were carried out on the metal-free, tin, and lead phthalocyanine molecules, whereas for copper phthalocyanine, restricted-spin open-shell HartreeFock (ROHF) calculations were performed. Semiempirical calculations with the PM3 Hamiltonian were also carried out for the metal-free and lead phthalocyanine molecules, as well as for their dimers. The STO3G [14,15], 3-21G [16], 6-31G(d) [17-20], and $6-31 \mathrm{G}(\mathrm{d}, \mathrm{p})$ basis sets were used in the HartreeFock calculations of metal-free phthalocyanine, and the copper phthalocyanine calculations were carried out using the basis of Stevens, Basch, and Krauss (SBK) [21-23], which replaces the core electrons with an effective core potential (ECP) and is doublezeta for the valence electrons. The $\operatorname{SBK}(d)$ basis was also used in the Hartree-Fock calculations of $\mathrm{CuPc}$ and SnPc. The lead phthalocyanine calculations were carried out with the $6-31 \mathrm{G}(\mathrm{d}, \mathrm{p})$ basis for all the atoms except lead, for which the SBK basis was used. All the DFT calculations were carried out with the DZP basis using the local density approximation (LDA).

The effects of solvation on $\mathrm{SnPc}$ were investigated through the use of continuum dielectric models. The SCRF [24-31] method available in the GAMESS program places the molecule in a spherical cavity surrounded by a continuum solvent of a given dielectric constant. The solute molecule induces a dipole in the solvent, which then interacts with the solute molecule. The reaction field method is also used to include solvation effects in the DFT calculations through the use of the COSMO $[32,33]$ program in DMOL. As in the GAMESS/SCRF program, COSMO places the solute molecule in a cavity surrounded by a dielectric continuum, but instead of using a spherical cavity, the van der

\footnotetext{
${ }^{1} \mathrm{DMol}$ is distributed by MSI/Biosym, Inc.
} 
Waals radii of solute atoms are used to generate a cavity having the same shape as the solute molecule. A second term is included in the free energy of solvation by COSMO in order to take into account non-electrostatic contributions due to dispersion and cavity formation effects; it is a linear function of solvent surface area and has been fit to data from linear-chain alkanes. In order to calculate the energies of excited states as well as transition dipole moments between states, to be used to compute oscillator strengths, the ZINDO [34] program was used, applying the INDO/S semiempirical Hamiltonian and CI with singly excited configurations.

\section{Results and discussion}

\subsection{Structure and energetics}

For the metal-free phthalocyanine, a stationary point with $D_{2 \mathrm{~h}}$ symmetry was found in the HartreeFock calculations with both the STO-3G and 3-21G basis sets, proving to be a saddle point having one imaginary frequency with a value of $2079 \mathrm{i} \mathrm{cm}^{-1}$ using the STO-3G basis and $1290 \mathrm{~cm}^{-1}$ using the 3-21G basis. The structure of this saddle point and the components of the mode corresponding to the imaginary frequency are illustrated in Fig. 1. With

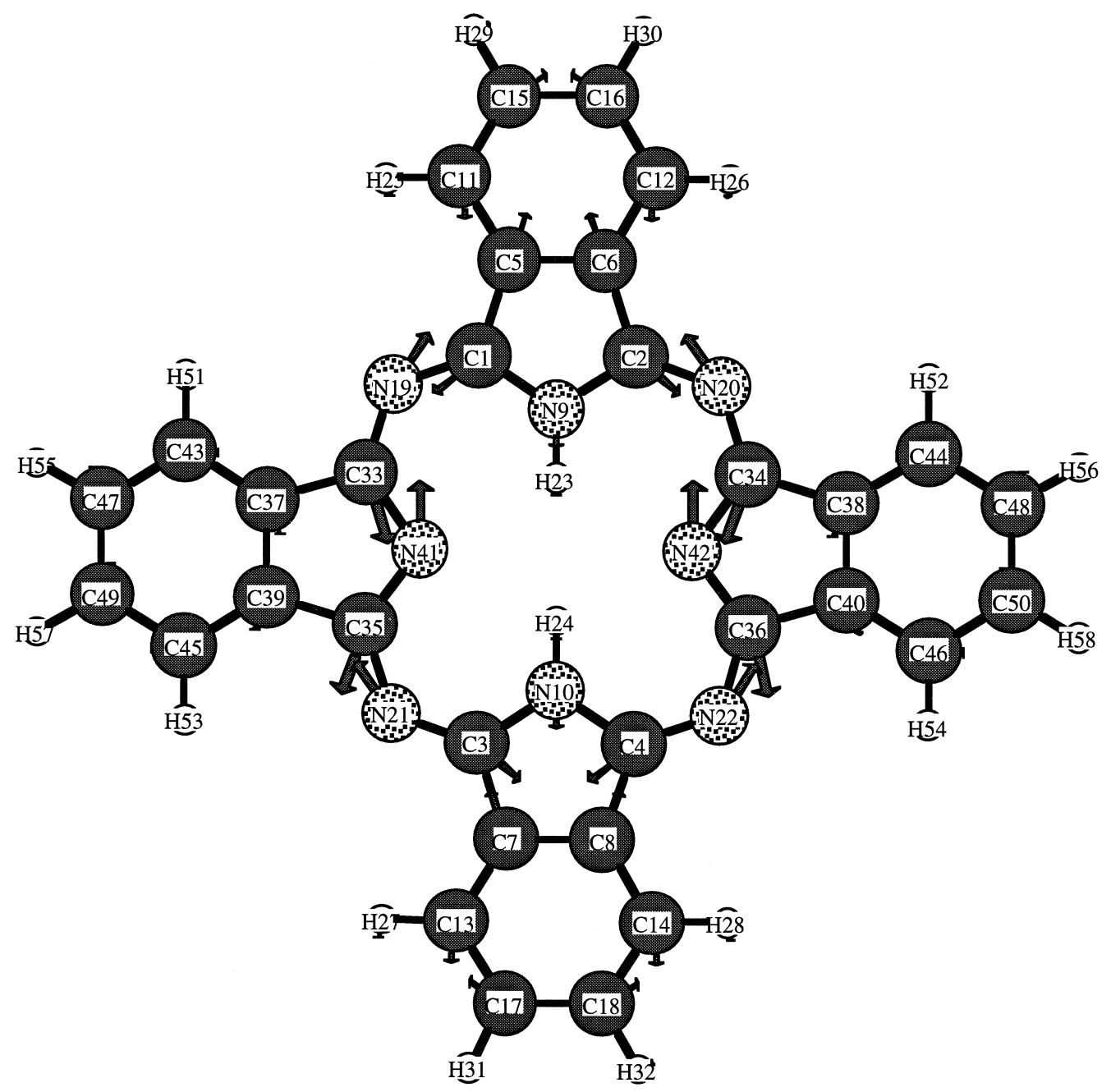

Fig. 1. The $D_{2 \mathrm{~h}}$ saddle point geometry for the metal-free phthalocyanine molecule. The components of the normal mode corresponding to the single imaginary frequency are indicated (the mode has no components out of the plane of the molecule). 
the use of the IRC method in GAMESS, a true minimum was found with $C_{2 \mathrm{v}}$ symmetry (note that two equivalent mirror image minima were found by proceeding downhill in both directions from the saddle-point). The symmetry axis for this geometry is in the plane of the molecule along the two $\mathrm{N}-\mathrm{H}$ bonds, rather than perpendicular to the plane of the molecule as for the $D_{2 \mathrm{~h}}$ geometry. This minimum was found at the Hartree-Fock level with four different basis sets: STO-3G, 321G, 6-31G(d), and 6-31G(d,p). The energy barrier from the Hartree-Fock minimum to the $D_{2 \mathrm{~h}}$ saddle point is $12.8 \mathrm{kcal} \mathrm{mol}^{-1}$ using the STO-3G basis, but only $3.7 \mathrm{kcal} \mathrm{mol}^{-1}$ using the 3-21G basis. The latter number is reduced to $1.4 \mathrm{kcal} \mathrm{mol}^{-1}$ when zero-point vibrational effects were included. The $6-31 \mathrm{G}(\mathrm{d}, \mathrm{p})$ structure is shown from two different perspectives in Fig. 2. Semiempirical calculations utilizing the PM3 Hamiltonian were carried out on the free-base phthalocyanine, since at this level of theory it was feasible to calculate the equilibrium geometry of the dimer, $\left(\mathrm{H}_{2} \mathrm{Pc}\right)_{2}$, which has the structure shown from two perspectives in Fig. 3. The calculated intermolecular interaction energy is $30.3 \mathrm{kcal} \mathrm{mol}^{-1}$.

The free-base phthalocyanine geometry was also optimized by DFT using the LDA. The bond lengths
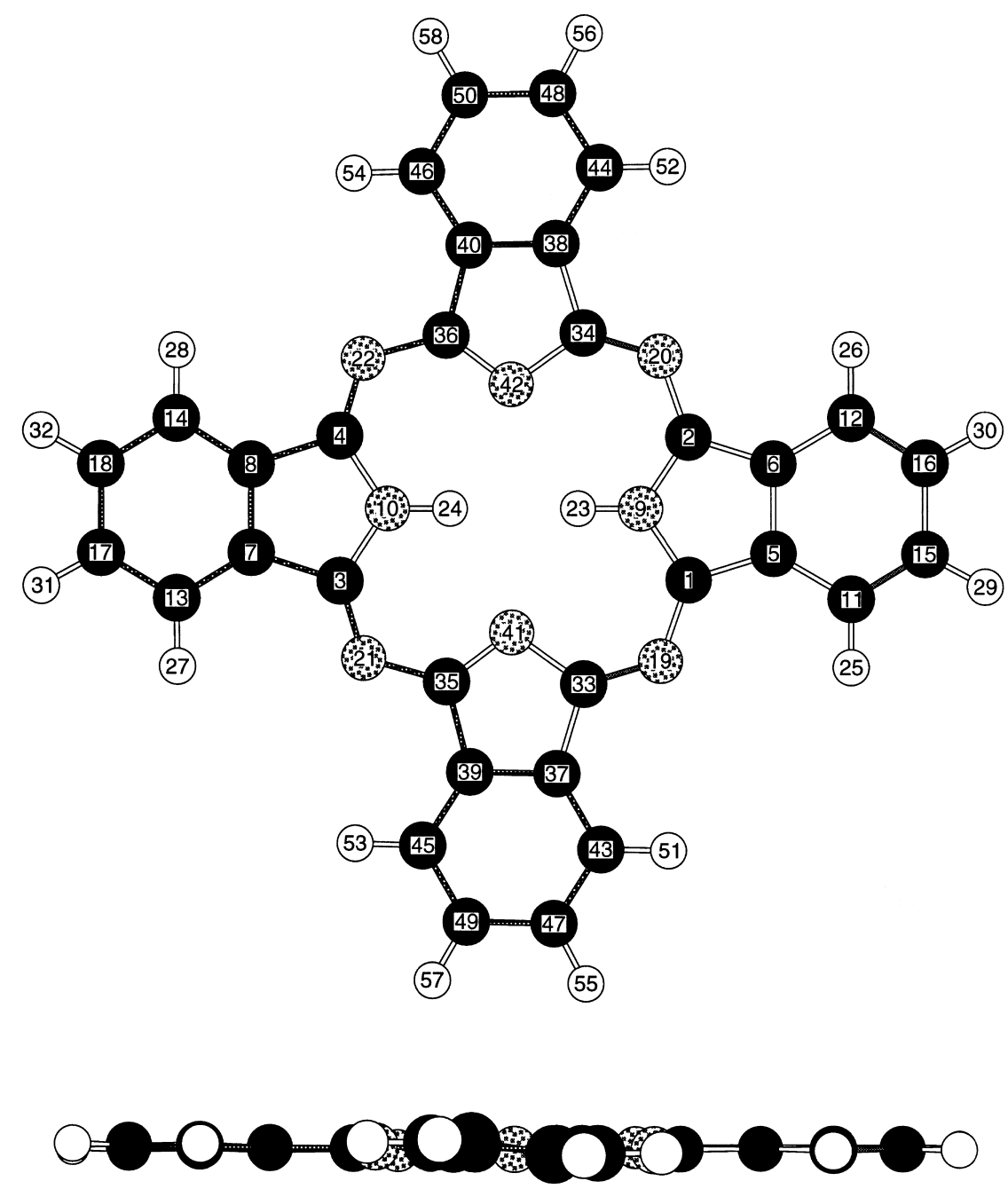

Fig. 2. The structure of the $C_{2 \mathrm{v}}$ minimum found for metal-free phthalocyanine. The side view illustrates that the structure is planar. 

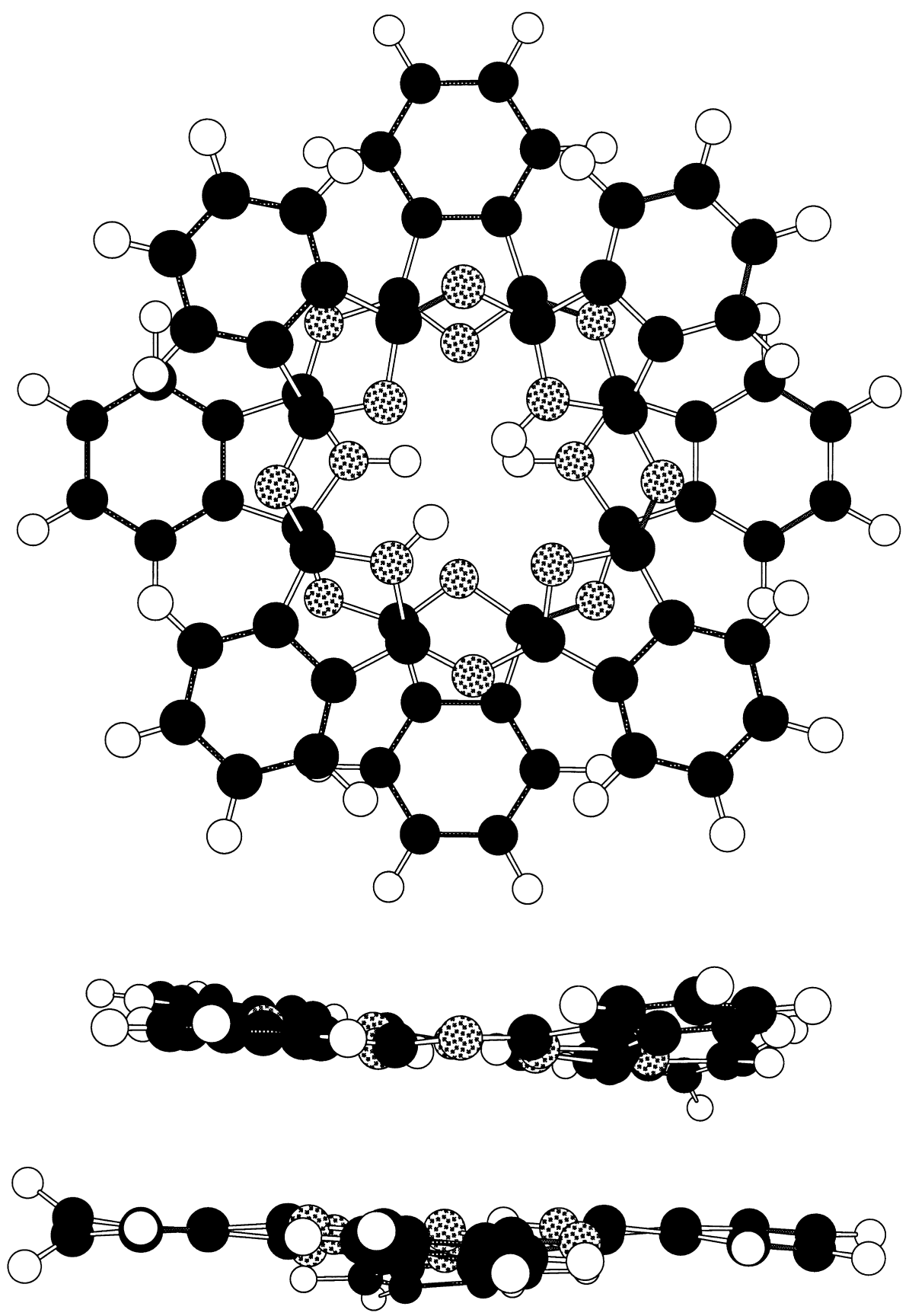

Fig. 3. The dimer structure of the metal-free phthalocyanine, as calculated at the semiempirical PM3 level of theory: (a) top view; (b) side view.

from the PM3 calculation, from the best HartreeFock calculation with the $631 \mathrm{G}(\mathrm{d}, \mathrm{p})$ basis, from the DFT calculation, and from experiment [10] are listed in Table 1, grouped according to equivalent symmetry. The average bond length in each group, as well as the standard deviation from this symmetryaveraged bond length, show that the DFT structure is closer to the expected $D_{2 \mathrm{~h}}$ symmetry than either the 
Table 1

Calculated bond lengths for the free-base phthalocyanine, grouped by equivalent bonds in $D_{2 \mathrm{~h}}$ symmetry; atom numbers are as given in Fig. 1

\begin{tabular}{|c|c|c|c|c|}
\hline Bond & PM3 (̊) & $6-31 G(\mathrm{~d}, \mathrm{p})(\AA)$ & LDA $(\AA)$ & Experiment $(\AA)$ \\
\hline N9-H23 & 0.996 & 0.991 & 1.035 & 0.930 \\
\hline $\mathrm{N} 10-\mathrm{H} 24$ & 1.002 & 0.994 & 1.035 & 0.950 \\
\hline $\mathrm{N} 9-\mathrm{C} 1$ & 1.397 & 1.354 & 1.367 & 1.380 \\
\hline N9-C2 & 1.397 & 1.354 & 1.367 & 1.360 \\
\hline $\mathrm{N} 10-\mathrm{C} 3$ & 1.428 & 1.368 & 1.368 & 1.380 \\
\hline $\mathrm{N} 10-\mathrm{C} 4$ & 1.428 & 1.368 & 1.368 & 1.360 \\
\hline N41-C33 & 1.453 & 1.400 & 1.362 & 1.380 \\
\hline $\mathrm{N} 41-\mathrm{C} 35$ & 1.333 & 1.294 & 1.365 & 1.370 \\
\hline N42-C34 & 1.453 & 1.400 & 1.362 & 1.380 \\
\hline $\mathrm{N} 42-\mathrm{C} 36$ & 1.333 & 1.294 & 1.365 & 1.370 \\
\hline $\mathrm{C} 1-\mathrm{C} 5$ & 1.431 & 1.403 & 1.436 & 1.470 \\
\hline $\mathrm{C} 2-\mathrm{C} 6$ & 1.431 & 1.404 & 1.436 & 1.460 \\
\hline $\mathrm{C} 3-\mathrm{C} 7$ & 1.478 & 1.475 & 1.437 & 1.470 \\
\hline $\mathrm{C} 4-\mathrm{C} 8$ & 1.478 & 1.475 & 1.437 & 1.460 \\
\hline C33-C37 & 1.476 & 1.474 & 1.447 & 1.490 \\
\hline C35-C39 & 1.475 & 1.472 & 1.451 & 1.460 \\
\hline C34-C38 & 1.476 & 1.474 & 1.447 & 1.490 \\
\hline C36-C40 & 1.475 & 1.472 & 1.451 & 1.460 \\
\hline C5-C6 & 1.427 & 1.414 & 1.402 & 1.400 \\
\hline $\mathrm{C} 7-\mathrm{C} 8$ & 1.419 & 1.385 & 1.404 & 1.400 \\
\hline C37-C39 & 1.422 & 1.384 & 1.395 & 1.410 \\
\hline $\mathrm{C} 38-\mathrm{C} 40$ & 1.422 & 1.384 & 1.395 & 1.410 \\
\hline $\mathrm{C} 5-\mathrm{C} 11$ & 1.408 & 1.418 & 1.385 & 1.390 \\
\hline C6-C12 & 1.408 & 1.418 & 1.385 & 1.390 \\
\hline $\mathrm{C} 7-\mathrm{C} 13$ & 1.380 & 1.380 & 1.386 & 1.390 \\
\hline C8-C14 & 1.380 & 1.380 & 1.386 & 1.390 \\
\hline C37-C43 & 1.381 & 1.382 & 1.383 & 1.370 \\
\hline C39-C45 & 1.381 & 1.383 & 1.383 & 1.400 \\
\hline C38-C44 & 1.381 & 1.382 & 1.383 & 1.370 \\
\hline $\mathrm{C} 40-\mathrm{C} 46$ & 1.381 & 1.383 & 1.383 & 1.400 \\
\hline $\mathrm{C} 11-\mathrm{C} 15$ & 1.374 & 1.356 & 1.384 & 1.390 \\
\hline $\mathrm{C} 12-\mathrm{C} 16$ & 1.374 & 1.356 & 1.384 & 1.380 \\
\hline C13-C17 & 1.400 & 1.386 & 1.384 & 1.390 \\
\hline C14-C18 & 1.400 & 1.386 & 1.384 & 1.380 \\
\hline $\mathrm{C} 43-\mathrm{C} 47$ & 1.400 & 1.386 & 1.389 & 1.410 \\
\hline $\mathrm{C} 45-\mathrm{C} 49$ & 1.400 & 1.385 & 1.389 & 1.380 \\
\hline C44-C48 & 1.400 & 1.386 & 1.389 & 1.410 \\
\hline $\mathrm{C} 46-\mathrm{C} 50$ & 1.400 & 1.385 & 1.389 & 1.380 \\
\hline $\mathrm{C} 15-\mathrm{C} 16$ & 1.416 & 1.431 & 1.400 & 1.420 \\
\hline C17-C18 & 1.388 & 1.393 & 1.400 & 1.420 \\
\hline
\end{tabular}


Table 1 (Continued)

\begin{tabular}{lllll}
\hline Bond & PM3 $(\AA)$ & $6-31 \mathrm{G}(\mathrm{d}, \mathrm{p})(\AA)$ & LDA $(\AA)$ & Experiment $(\AA)$ \\
\hline C47-C49 & 1.390 & 1.395 & 1.396 & 1.430 \\
C48-C50 & 1.390 & 1.395 & 1.396 & 1.430 \\
C1-N19 & & & 1.309 & 1.310 \\
C2-N20 & 1.392 & 1.351 & 1.309 & 1.340 \\
C3-N21 & 1.392 & 1.351 & 1.311 & 1.310 \\
C4-N22 & 1.311 & 1.273 & 1.311 & 1.340 \\
C33-N19 & 1.311 & 1.273 & & 1.326 \\
C35-N21 & & & 1.326 & 1.310 \\
C34-N20 & 1.300 & 1.271 & 1.326 & 1.300 \\
C36-N22 & 1.403 & 1.368 & 1.326 & 1.310 \\
\hline
\end{tabular}

PM3, Hartree-Fock or experimental structures. The symmetry-averaged bond lengths are used to compare the Hartree-Fock and DFT geometries with the experimental geometry in Fig. 4. The error bars indicate the standard deviation from the expected $D_{2 \mathrm{~h}}$ symmetry. The error bars for some points, mainly from the DFT calculation, are not observed because they are very small. The large error bars for the

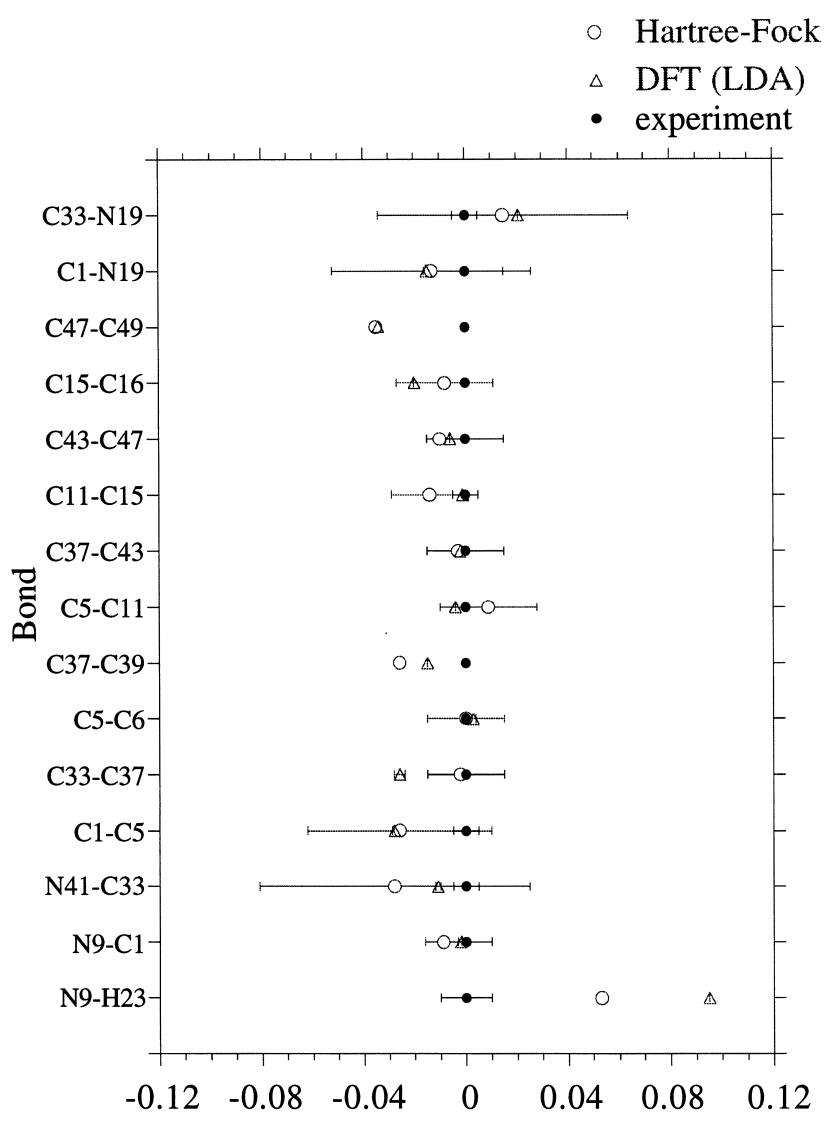

Difference from experimental geometry (angstroms)

Fig. 4. Bond length comparison for free-base phthalocyanine. Error bars represent deviation from $D_{2 \mathrm{~h}}$ symmetry. 

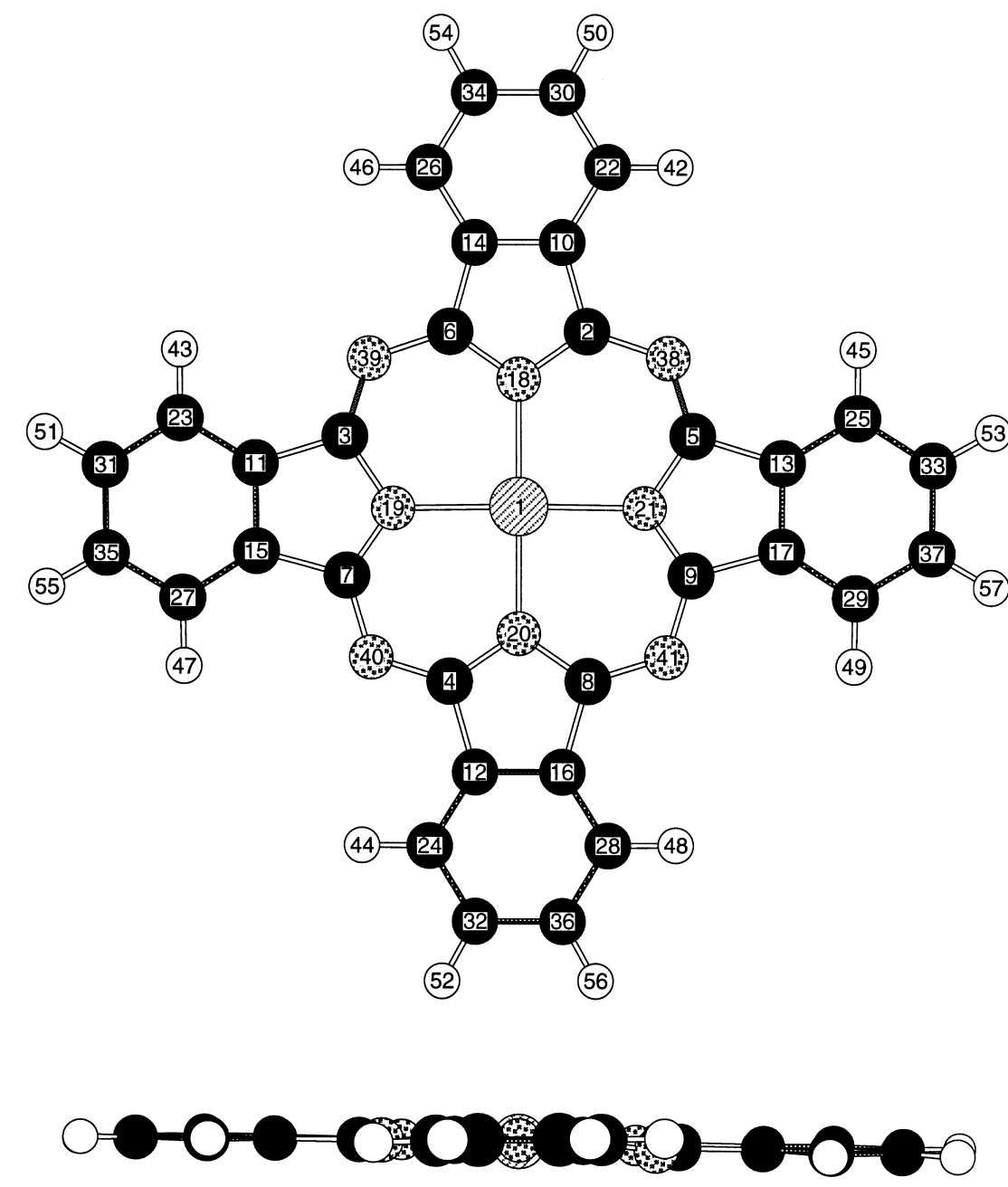

Fig. 5. The structure of the $C_{2 \mathrm{v}}$ minimum found for copper phthalocyanine. The side view illustrates that the structure is planar.

Hartree-Fock results, indicating a large deviation from the expected symmetry, are attributed to the neglect of electron correlation in the single determinant Hartree-Fock model, which does not allow for sufficient delocalization of the $\mathrm{p}$ orbitals. This explanation is consistent with the results of previous calculations on porphyrins [35], where it was found that including electron correlation at the MP2 level of theory regained the expected symmetry. The deviation of the experimental structure from the $D_{2 \mathrm{~h}}$ symmetry can be attributed to neighboring molecule effects in the crystal lattice. The agreement between the calculated and experimental bond lengths is good for both theoretical methods, except for the bond length from a pyrrole nitrogen to a central hydrogen, where the calculated bond length is significantly longer than the experimental one. However, the neutron diffraction study of this structure indicates a nearly $D_{4 \mathrm{~h}}$ structure with "four well defined halfhydrogen atoms, one associated with each of the pyrrole nitrogen atoms" [10]. Apparently, the rate of $\mathrm{H}$-transfer between the pyrrole nitrogen atoms is so fast that the experimental method cannot differentiate between the pyrrole nitrogen atoms that are bonded to a hydrogen atom and those that are not. Since the computations result in bond lengths to each of two full hydrogen atoms, they are significantly longer than the bond lengths given in Ref. [10]. 
- Hartree-Fock SBK(d)

$\triangle$ DFT (LDA)

- Experiment

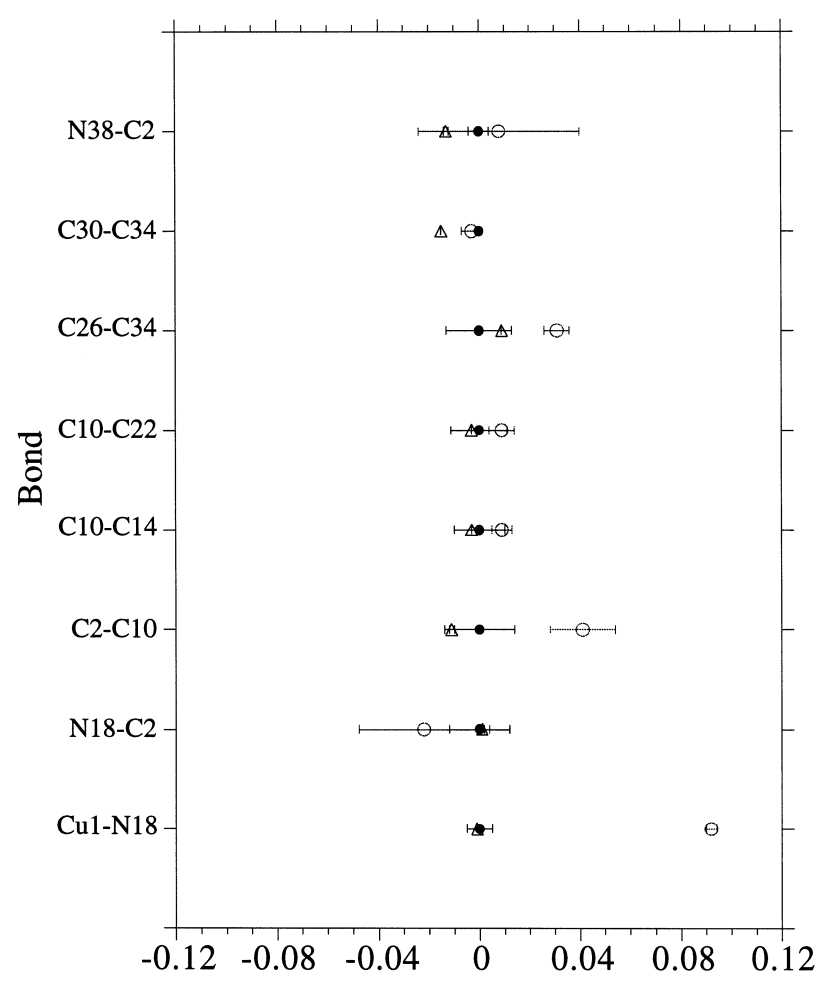

Difference from experimental geometry (angstroms)

Fig. 6. Bond length comparison for copper phthalocyanine. Error bars represent deviation from $D_{4 \mathrm{~h}}$ symmetry.

A planar structure with $D_{4 \mathrm{~h}}$ symmetry was expected for copper phthalocyanine. In Hartree-Fock calculations with the SBK basis set, a stationary point with $D_{4 \mathrm{~h}}$ symmetry was located, and a Hessian, or energysecond-derivative, calculation was carried out in order to characterize the stationary point and obtain the vibrational frequencies. This structure was found to have two degenerate modes with imaginary frequencies of $1968.58 \mathrm{im}^{-1}$, thus being a second-order saddle point and not a minimum. Using the IRC following methods in GAMESS, a minimum was located which was $78 \mathrm{kcal} \mathrm{mol}^{-1}$ lower in energy. This minimum energy structure, shown in Fig. 5, is planar with $C_{2 \mathrm{v}}$ symmetry, although its deviations from $D_{4 \mathrm{~h}}$ symmetry are small. By following each of the two degenerate modes downhill in both the positive and negative directions, four equivalent minima were found.

On the other hand, the DFT calculations resulted in a minimum energy structure that is very close to the expected $D_{4 \mathrm{~h}}$ symmetry. As was the case with the freebase phthalocyanine calculations, this is attributed to the inclusion of electron correlation in DFT, whereas the neglect of electron correlation in Hartree-Fock theory may explain its failure to predict this high symmetry equilibrium geometry. Fig. 6 shows the difference in the symmetry-averaged bond lengths from the experimental bond lengths [6]. As in Fig. 4, the error bars indicate the standard deviation from the expected symmetry. The Hartree-Fock equilibrium structure, with only $C_{2 v}$ symmetry, has the highest deviation from the expected $D_{4 \mathrm{~h}}$ symmetry. The 


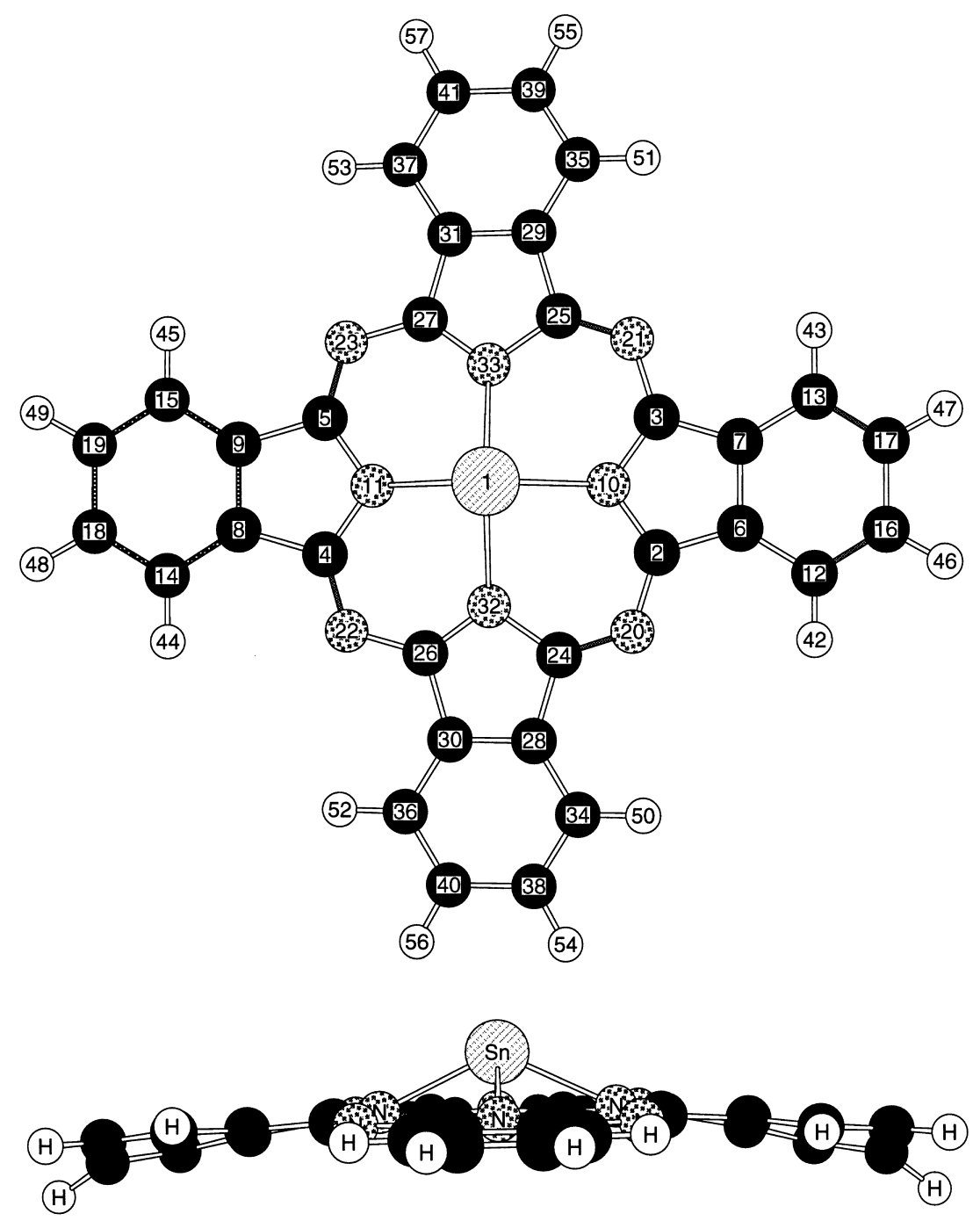

Fig. 7. The structure of the $C_{\mathrm{s}}$ minimum found for tin phthalocyanine. The side view illustrates that the structure is non-planar.

symmetry-averaged bond lengths from both calculations agree fairly well with experiment, with the exception of the $\mathrm{Cu}-\mathrm{N}$ bond in the Hartree-Fock calculation, which is $0.09 \AA$ longer than that found in the experimental structure. The DFT calculation, on the other hand, agrees closely with the experimental value for this bond length. The DFT bond lengths, with an average difference from experiment of $0.007 \AA$, are in general closer to the $\mathrm{X}$-ray crystal structure than those from the Hartree-Fock calculations, which have an average difference of $0.027 \AA$. Even if the $\mathrm{Cu}-\mathrm{N}$ bond is excluded from the average, the DFT results are still in better agreement with experiment, with an average difference of $0.008 \AA$ versus $0.017 \AA$ for the Hartree-Fock results.

The structure of tin phthalocyanine is non-planar, as shown both experimentally and computationally, with an expected symmetry of $C_{4 \mathrm{v}}$ rather than $D_{4 \mathrm{~h}}$. However, as was the case with $\mathrm{H}_{2} \mathrm{Pc}$ and $\mathrm{CuPc}$, the Hartree-Fock stationary point with the expected symmetry is not a minimum, but rather a second-order saddle-point. The Hartree-Fock minimum, which can be found by using IRC following from this saddle point, has only $C_{\mathrm{s}}$ symmetry, where the symmetry 
plane passes through the metal atom and two opposing pyrrole nitrogen atoms. This minimum energy geometry is shown from two perspectives in Fig. 7. As with the $\mathrm{CuPc}$, there are four equivalent minima.

The DFT calculations obtained, once again, a minimum energy configuration very close to the expected symmetry. In Fig. 8, the bond lengths obtained for $\mathrm{SnPc}$ in the two theoretical methods, HF and DFT, are compared with the experimental bond lengths obtained in the crystal structure study [7]. As expected, the error bars, used to show the standard deviation from the expected symmetry, are very small for the DFT calculations, but fairly large for the HF results. The symmetry-averaged bond lengths from both calculations agree fairly well with the experimental results, differing only by an average of $0.009 \AA$ in the DFT calculations and by an average of $0.014 \AA$ in the Hartree-Fock calculations.

Two stationary points for the lead phthalocyanine have been found at the Hartree-Fock level. The first stationary point, which is planar, was found not to be a minimum. This stationary point has $C_{2 \mathrm{v}}$ symmetry, similar to the Hartree-Fock minima found for $\mathrm{H}_{2} \mathrm{Pc}$ and $\mathrm{CuPc}$. A structure assumed to be a Hartree-Fock minimum has been found with an energy about $108 \mathrm{kcal} \mathrm{mol}^{-1}$ lower than that of the planar structure, shown in Fig. 9. This structure has $C_{\mathrm{s}}$ symmetry, similar to the Hartree-Fock minimum found for $\mathrm{SnPc}$. The minimum for $\mathrm{PbPc}$ is expected to have $C_{4 \mathrm{v}}$ symmetry, similar to $\mathrm{SnPc}$, but again the Hartree-Fock method does not obtain the correct symmetry owing to the neglect of electron correlation. Since the program we used for the DFT calculations, DMOL [13], does not have parameters for the lead atom, we were unable to carry out DFT calculations on lead phthalocyanine. However, in Fig. 10, we report a comparison of two X-ray crystal structure studies [8,9]. Whereas in Figs. 4, 6, and 8 the bond lengths comparison was carried out relative to the experimental bond lengths, in Fig. 10 the two experimental structures are compared with the Hartree-Fock calculation by using the calculated geometry as a reference. As before, the error bars indicate the standard deviation from the expected $C_{4 \mathrm{v}}$ geometry. The results listed for the monoclinic crystal structure [8] indicate nearly exact $C_{4 \mathrm{v}}$ symmetry, so that the error bars on these points are very small, averaging only $0.001 \AA$. The
- Hartree-Fock SBK(d)

$\triangle$ DFT (LDA)

- experiment

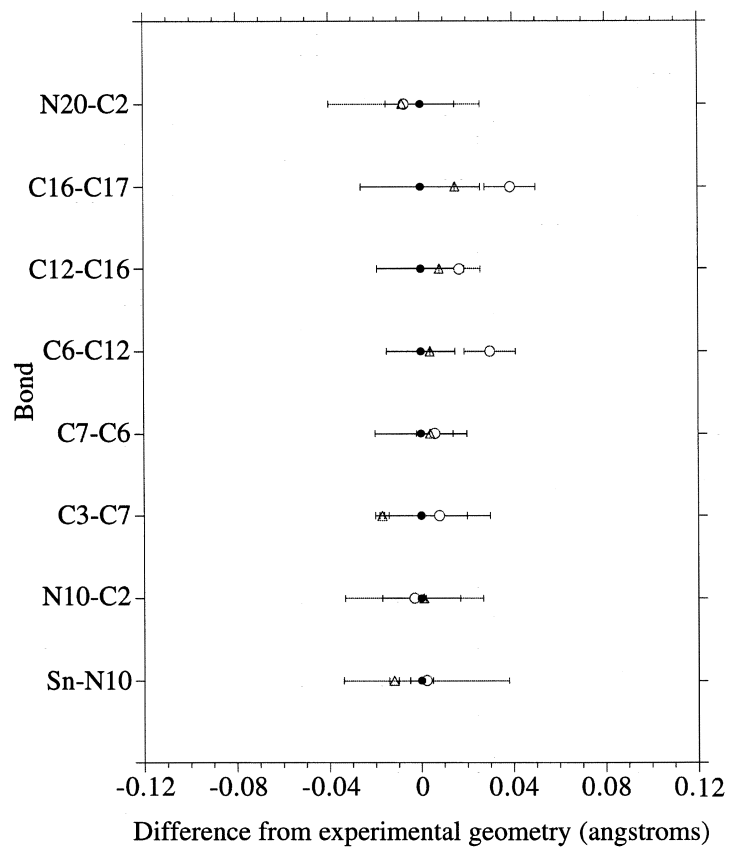

Fig. 8. Bond length comparison for tin phthalocyanine. Error bars represent deviation from $C_{4 \mathrm{v}}$ symmetry.

Hartree-Fock results have the largest error bars with an average standard deviation of $0.020 \AA$, whereas the average standard deviation of the triclinic crystal structure results [9] is $0.013 \AA$. The calculated bond lengths and those found in the triclinic crystal agree fairly well, with an average difference of only $0.016 \AA$, whereas the bond lengths from the monoclinic crystal differ from the calculated values by an average of $0.044 \AA$. This can be explained by the close packing in the monoclinic crystal, where the molecules are stacked in columns, as stacks of bowls. This close packing results in a larger interaction with neighboring molecules, and thus a larger deviation from the gas-phase equilibrium structure that we calculated. In particular, this close packing results in a strong repulsion between neighboring lead atoms, thus shortening the $\mathrm{Pb}-\mathrm{N}$ bonds considerably from what they are in the more loosely packed triclinic crystal or in the calculated gas-phase structure.

The structure of the lead phthalocyanine dimer as optimized at the PM3 semiempirical level of theory is 

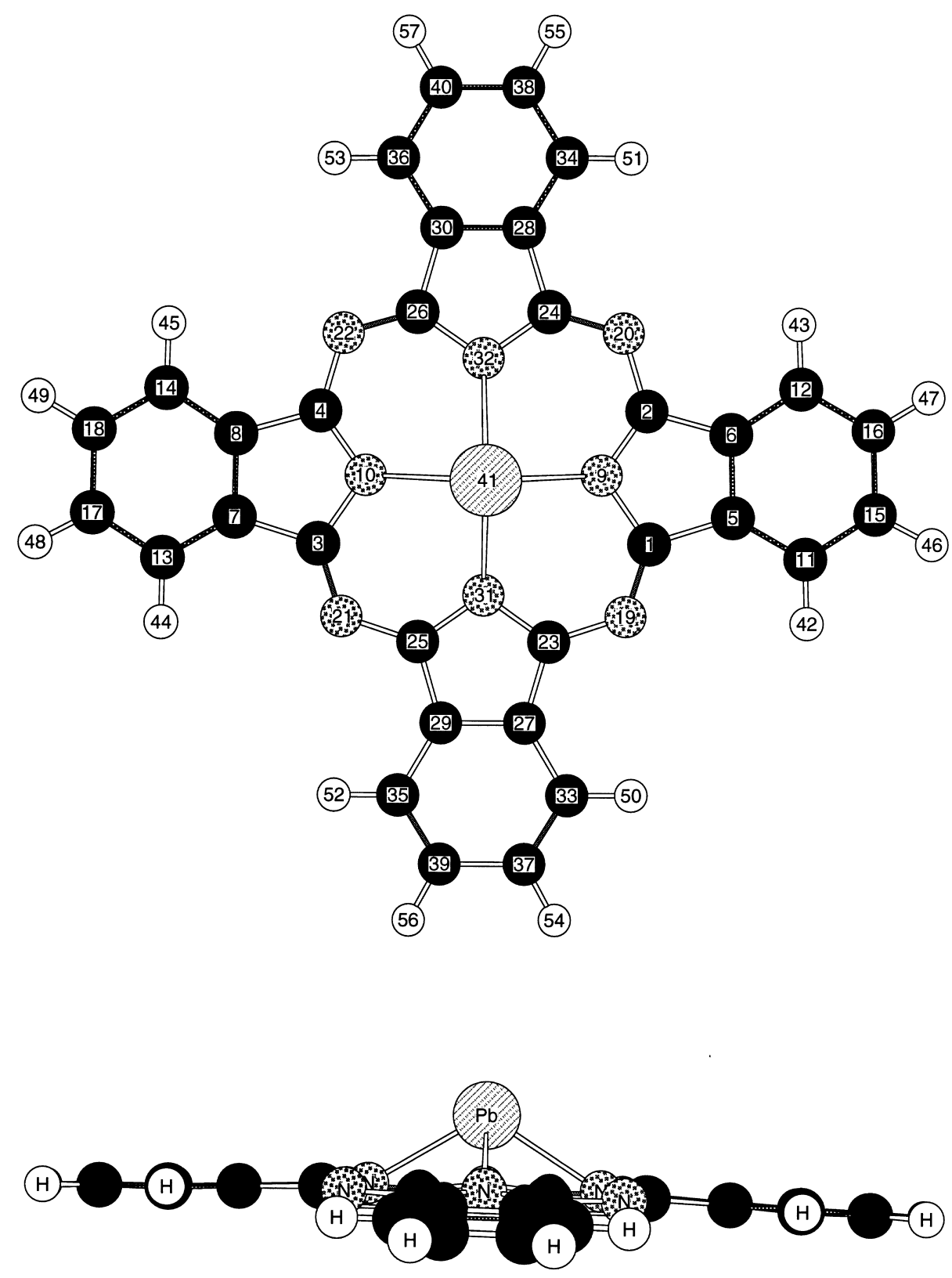

Fig. 9. The structure of the $C_{\mathrm{s}}$ minimum found for lead phthalocyanine. The side view illustrates that the structure is non-planar.

shown in Fig. 11 from two perspectives. The lead atoms are positioned as far from each other as possible, and the porphyrin rings are offset from each other by $45^{\circ}$. The calculated interaction energy at this level of theory is $36 \mathrm{kcal} \mathrm{mol}^{-1}$.

The effects of solvation on the calculated structure of $\mathrm{SnPc}$ are summarized in Fig. 12, which shows the change in the symmetry-averaged bond lengths due to the SCRF in the Hartree-Fock calculation and due to the COSMO model in the DFT calculation. For the SCRF calculation in GAMESS, a cavity radius of $6.03 \AA$ was used in order to simulate a cavity with approximately the same volume as the solute molecule, and a dielectric constant of 80 was used 


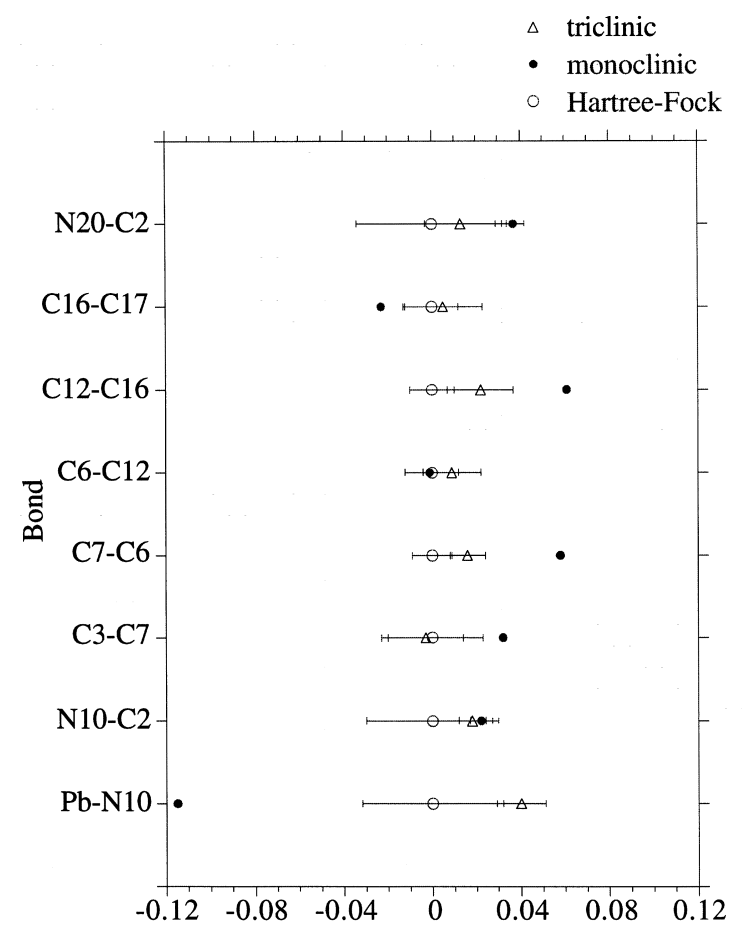

Difference from Hartree-Fock geometry (angstroms)

Fig. 10. Bond length comparison for lead phthalocyanine. Error bars represent deviation from $C_{4 \mathrm{v}}$ symmetry.

to simulate water. Although water may not be the primary solvent of interest for our studies of phthalocyanines, this dielectric constant was used to mimic the largest possible effect. In the SCRF calculation, the changes in the symmetry-averaged bond lengths, none of which have a magnitude of over $0.002 \AA$, are too small to be significant. Some of the individual bonds did change by a more significant amount; the largest change is in one of the $\mathrm{Sn}-\mathrm{N}$ bonds which increased in length by about $0.01 \AA$, while the opposite bond decreased in length by about the same amount. The stabilization energy gained by the SCRF solvation model is about $2.5 \mathrm{kcal} \mathrm{mol}^{-1}$. The DFT/COSMO program used a dielectric constant of 78.54 by default to simulate water. As can be seen in Fig. 12, there were significant changes in the symmetry-averaged bond lengths for two of the symmetry-unique bonds, particularly for the $\mathrm{Sn}-\mathrm{N}$ bond, which increased by an average of $0.029 \AA$. This effect was expected, as the tin atom's out-ofplane position is the main source of polarity in this molecule, and the effect of the solvent field is to further polarize the molecule, pulling the tin atom farther out of the phthalocyanine plane. The total solvation energy calculated in the DFT/COSMO program was $22.3 \mathrm{kcal} \mathrm{mol}^{-1}, \quad 14.8 \mathrm{kcal} \mathrm{mol}^{-1}$ of which was the electrostatic contribution. The much smaller solvation energy calculated by the HartreeFock/SCRF method could be because the SCRF method in GAMESS uses only induced dipoles, and it may be that higher-order induced multipoles are needed in order to calculate the correct solvation energy.

\subsection{Spectra}

Vibrational spectra for the free-base phthalocyanine were calculated in the harmonic approximation using Hartree-Fock theory. In Fig. 13, the calculated vibrational frequencies and intensities for free-base phthalocyanine are compared with the spectra observed by Kobayashi [36]. The frequencies were calculated at the Hartree-Fock level using the 3-21G basis set at the minimum-energy geometry obtained at this level of theory, and were scaled by the factor 0.89 , as is common for Hartree-Fock frequencies in order to adjust for the errors introduced by the harmonic approximation and by the singledeterminant HF theory. The intensities of the vibrational frequencies are calculated in the GAMESS program by projecting the dipole derivative tensor onto the normal mode vector, and squaring the result. The experimental intensities are approximate since they were derived only from a plot. For the largest six frequencies, no intensities were available; therefore, for comparison purposes, the intensity for each of these frequencies was set to that of the nearest calculated frequency. Some of the calculated frequencies agree relatively well with experiment, particularly for the observed frequencies above $400 \mathrm{~cm}^{-1}$. However, the calculation shows additional frequencies above $400 \mathrm{~cm}^{-1}$ with significant intensities that are not part of the observed spectrum. Also, the observed spectrum has strong peaks at $269 \mathrm{~cm}^{-1}$, $282 \mathrm{~cm}^{-1}$, and $342 \mathrm{~cm}^{-1}$ that do not show in the calculated vibrational frequencies.

Electronic spectra in the visible band were calculated for the free-base phthalocyanine using the ZINDO program, which carries out configuration interaction 
with the INDO semiempirical Hamiltonian. This calculation was carried out for each of four geometries for free-base phthalocyanine: the ab initio HartreeFock geometry of Fig. 2, the DFT geometry obtained using LDA, the experimental geometry of Ref. [10], and one obtained in a semiempirical PM3 optimization. The calculated spectra obtained for the transitions from the ground state to the first 19 excited states are listed in Table 2, where they are compared with measured spectra [37,38] in the gas-phase and in 1-chloronaphthalene solution. The calculated oscillator strength for each transition, which is listed in parentheses next to the corresponding wavelength, was obtained from

$f_{\mathrm{na}}=\frac{8 \pi^{2} m_{\mathrm{e}} \nu_{\mathrm{na}}\left|\mu_{\mathrm{na}}\right|^{2}}{3 h e^{2}}$

where $m_{\mathrm{e}}$ and $e$ are the electronic mass and charge respectively, $h$ is Planck's constant, $\nu_{\text {na }}$ is the frequency of the a $\rightarrow \mathrm{n}$ transition and $\mu_{\mathrm{na}}$ is the corresponding transition dipole moment:

$\mu=\left\langle\Psi_{\mathrm{n}}|e r| \Psi_{\mathrm{a}}\right\rangle$

The oscillator strengths for the measured spectra were calculated from

$f_{\text {na }}=\frac{4 m_{\mathrm{e}} c \varepsilon_{0} \ln 10}{N_{\mathrm{A}} e^{2}} A_{\text {na }}$

where $c$ is the speed of light in a vacuum, $\varepsilon_{0}$ is the permittivity of free space, $N_{\mathrm{A}}$ is Avogadro's number, and $A_{\text {na }}$ is the area under the spectral line

$A_{\text {na }}=\int \varepsilon_{\text {na }} \mathrm{d} \nu$

and $\varepsilon_{\text {na }}$ is the molar extinction coefficient. This area was evaluated for each transition by measuring the width of each peak in Fig. 4 of Ref. [38], transforming the $\varepsilon_{\text {na }}$ from Ref. [37] to a function of frequency instead of wavelength, and carrying out the integration assuming a Gaussian functional form. The

Table 2

The electronic spectra of free-base phthalocyanine, calculated using the ZINDO program at four different geometries, three of which are computationally optimized geometries with the fourth being the experimental geometry [10]. Two sets of measured spectra are also listed, one measured in the vapor phase and the other in the solvent 1-chloronaphthalene $(\mathrm{CN})$. Oscillator strengths are given in parentheses.

\begin{tabular}{|c|c|c|c|c|c|c|c|c|c|c|}
\hline \multicolumn{2}{|c|}{$\begin{array}{l}\text { ZINDO//LDA } \\
\text { geometry }\end{array}$} & \multicolumn{2}{|c|}{$\begin{array}{l}\text { ZINDO//HF/6-31G** } \\
\text { geometry }\end{array}$} & \multicolumn{2}{|c|}{$\begin{array}{l}\text { ZINDO//PM3 } \\
\text { geometry }\end{array}$} & \multicolumn{2}{|c|}{$\begin{array}{l}\text { ZINDO//experimental } \\
\text { geometry }\end{array}$} & \multicolumn{2}{|c|}{ Measured in $\mathrm{CN}$} & \multirow{3}{*}{$\begin{array}{l}\begin{array}{l}\text { Measured } \\
\text { vapor }\end{array} \\
686\end{array}$} \\
\hline 727 & $(0.82)$ & 601 & $(0.71)$ & 637 & $(0.75)$ & 756 & $(0.78)$ & 700 & $(0.22)$ & \\
\hline & & & & & & 665 & $(0.51)$ & & & \\
\hline \multirow[t]{3}{*}{684} & $(0.89)$ & 586 & (0.69) & 618 & $(0.67)$ & 707 & (0.77) & 638 & $(0.18)$ & 622 \\
\hline & & & & & & 602 & $(0.13)$ & & & \\
\hline & & & & & & 554 & $(0.02)$ & & & \\
\hline 413 & $(0.00)$ & 375 & $(0.12)$ & 393 & $(0.12)$ & 424 & $(0.02)$ & & & \\
\hline 398 & $(0.00)$ & 359 & $(0.08)$ & 377 & $(0.09)$ & 415 & $(0.00)$ & & & \\
\hline 360 & $(0.05)$ & 324 & $(0.09)$ & 337 & $(0.12)$ & 391 & $(0.39)$ & & & \\
\hline 351 & $(0.61)$ & 316 & $(0.14)$ & 332 & $(0.25)$ & 368 & (0.09) & & & 340 \\
\hline 340 & $(0.00)$ & 314 & $(0.00)$ & 326 & $(0.04)$ & 355 & $(0.29)$ & & & \\
\hline 336 & $(0.14)$ & 312 & $(0.07)$ & 318 & $(0.02)$ & 350 & $(0.51)$ & & & \\
\hline 331 & $(0.00)$ & 310 & $(0.01)$ & 314 & $(0.00)$ & 339 & $(0.02)$ & & & \\
\hline 314 & (1.05) & 308 & $(0.16)$ & 304 & $(1.28)$ & 329 & $(0.01)$ & & & \\
\hline 301 & $(0.00)$ & 305 & $(0.02)$ & 299 & $(0.70)$ & 319 & $(0.51)$ & & & \\
\hline 293 & (1.66) & 292 & (1.34) & 286 & $(0.01)$ & 314 & (0.09) & & & \\
\hline 291 & $(0.00)$ & 291 & $(0.54)$ & 284 & $(0.13)$ & 306 & $(0.44)$ & & & \\
\hline 288 & (1.11) & 276 & $(0.12)$ & 280 & $(0.00)$ & 303 & $(0.63)$ & & & \\
\hline 285 & (1.63) & 276 & $(0.00)$ & 278 & $(0.12)$ & 302 & $(0.22)$ & & & \\
\hline 280 & $(0.00)$ & 269 & $(0.76)$ & 273 & (1.47) & 300 & $(0.48)$ & & & \\
\hline 279 & $(0.00)$ & 269 & $(0.00)$ & 273 & $(0.05)$ & 292 & $(0.45)$ & & & \\
\hline 277 & $(0.01)$ & 262 & $(0.07)$ & 266 & $(0.04)$ & 290 & $(0.87)$ & & & \\
\hline 276 & $(0.00)$ & 259 & $(0.00)$ & 265 & $(0.06)$ & 288 & $(0.63)$ & & & \\
\hline
\end{tabular}



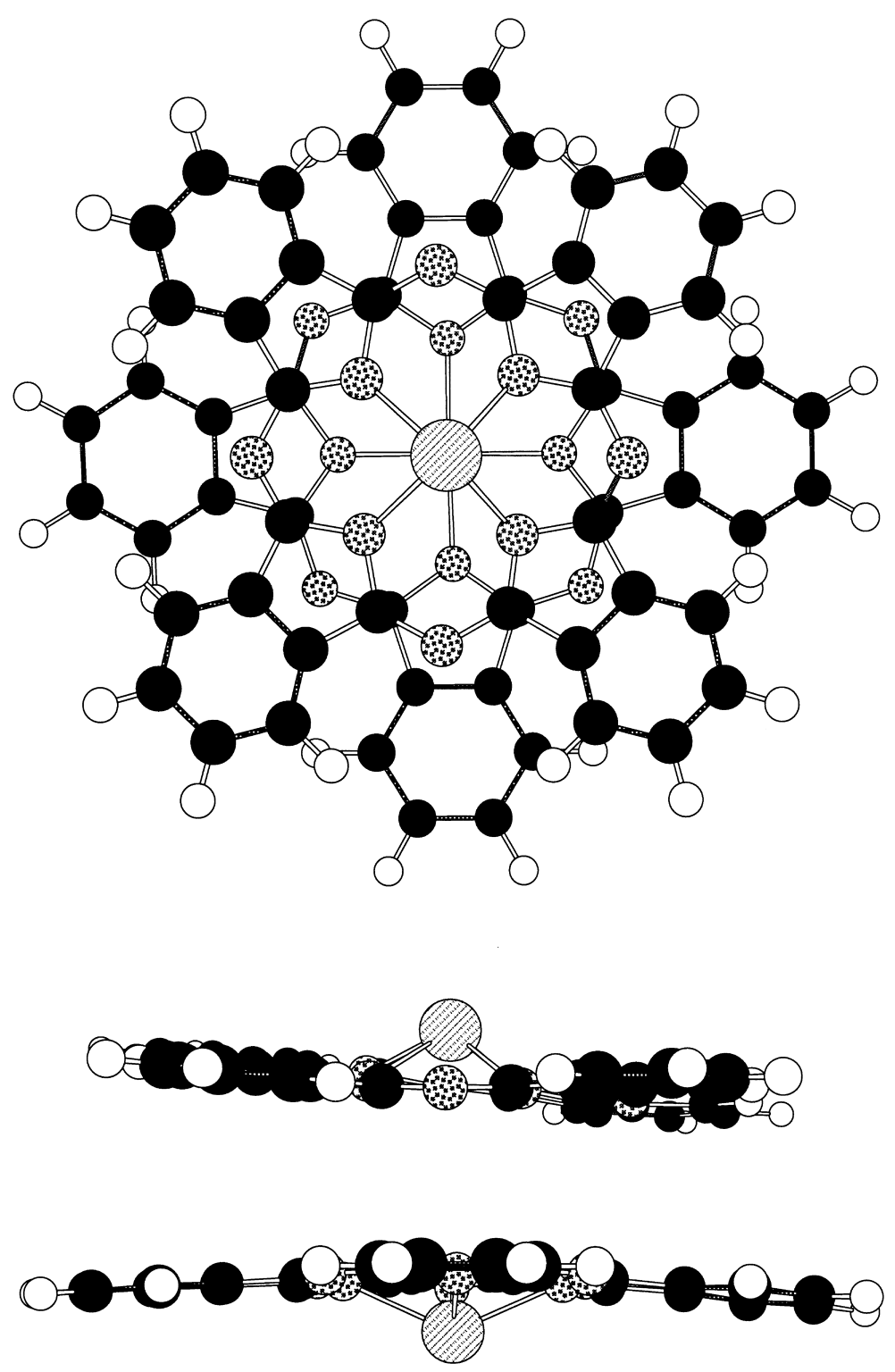

Fig. 11. The dimer structure of lead phthalocyanine, as calculated at the semiempirical PM3 level of theory: (a) top view; (b) side view.

intensities of the measured spectra are available only from the measurements made in solution.

The Q-band is split into two peaks in free-base phthalocyanine since it has only twofold symmetry rather than the fourfold symmetry of the metal phthalocyanines. This feature is seen in all four sets of calculated spectra as well as in the two sets of measured spectra, although in the solvated system the Q-band has been split into four or five peaks.
Since the calculated spectra are for an isolated molecule, the vapor-phase measurements are most applicable for a direct comparison. The calculated spectra show that this method is very sensitive to the geometry used. Although all the spectra were calculated at the same level of theory, the four different geometries caused variations in both directions from the experimental spectra, with an overall average error of $8 \%$. The spectra calculated 


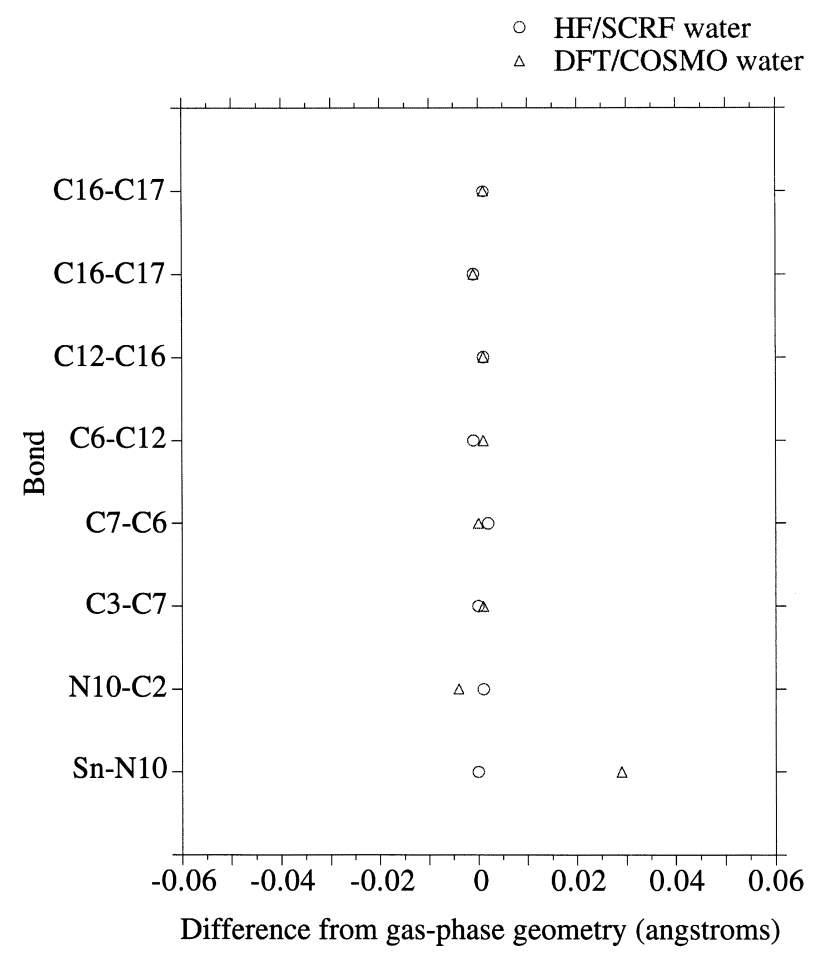

Fig. 12. The effect of solvation on the bond lengths in SnPc.

using the LDA and experimental geometries have longer wavelengths than the measured spectra, whereas those calculated using the HF and PM3 geometries have shorter wavelengths for the Q-band transitions. From the LDA geometry, the wavelengths of the first and second peaks are 6\% and $10 \%$ longer than measured, whereas those calculated from the experimental geometry are $10 \%$ and $14 \%$ longer than the measured wavelengths. From the HartreeFock geometry, the wavelengths of the first and second peaks are $12 \%$ and $6 \%$ shorter than the measured values, whereas from the PM3 geometry they are just $7 \%$ and $1 \%$ too short.

Although the calculations all consistently had two Q-band peaks with oscillator strengths in the $0.7-0.9$ range, identification of the B-band transition, which has been measured in the vapor-phase at $340 \mathrm{~nm}$, is more ambiguous. The calculation from the LDA geometry is the least deceptive, having a strong peak at $351 \mathrm{~nm}(f=0.61)$, and no other peaks nearly this strong up to $314 \mathrm{~nm}$, which is into the ultraviolet spectrum. The wavelength of $351 \mathrm{~nm}$ is only $3 \%$ longer than the measured spectral wavelength. The calculation at the Hartree-Fock geometry did not give a strong, distinct line for the B-band, but instead gave several weak lines. The lines at $375 \mathrm{~nm}$ $(f=0.12), 359 \mathrm{~nm}(f=0.08)$, and $324 \mathrm{~nm}(f=0.09)$ are the best candidates for this spectral line, whereas the slightly stronger line at $316 \mathrm{~nm}(f=0.14)$ is considered to be in the ultraviolet. This splitting of the B-band is attributed to the lower symmetry of the Hartree-Fock geometry. The calculations from the PM3 and experimental geometries also show some splitting of this band, but the line at $332 \mathrm{~nm}$ ( $f=0.35$ ) from the PM3 geometry and the line at $350 \mathrm{~nm}(f=0.51)$ from the experimental geometry are clearly the strongest lines in the B-band range. These lines agree with the experimental wavelength value to within $3 \%$.

The calculated spectra for the two Q-band lines indicate oscillator strengths in the range 0.7-0.9. None of the individual oscillator strengths calculated from the measured spectra in 1-chloronaphthalene are this high, although if we assume that the $\mathrm{Q}_{x}$ line has been split into the two lines at $700 \mathrm{~nm}$ and $665 \mathrm{~nm}$, the combined oscillator strength for this transition is 0.73 . 
- scaled Hartree-Fock

\ experiment

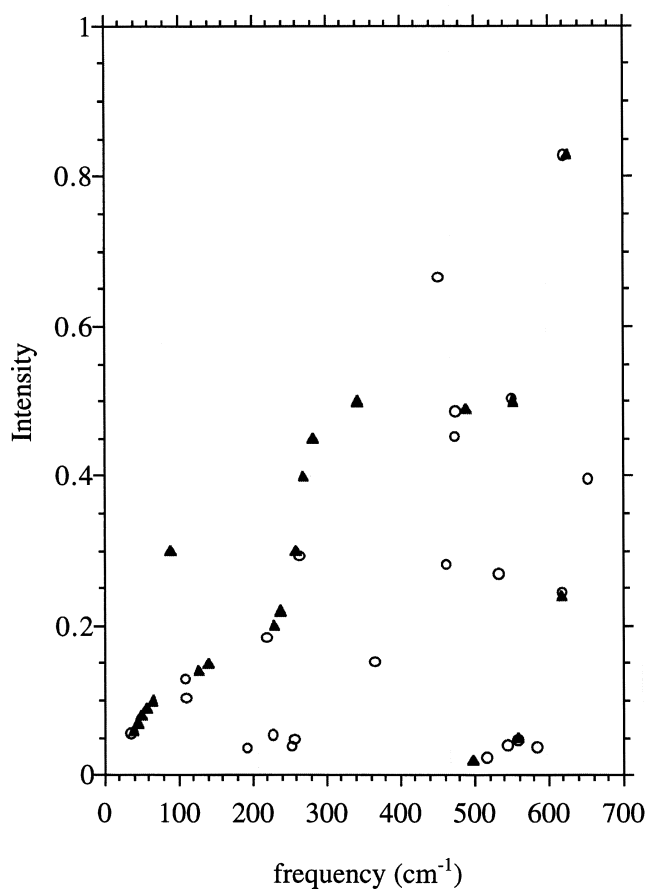

Fig. 13. The vibrational spectrum for free-base phthalocyanine. The Hartree-Fock spectra were obtained by using the harmonic approximation and scaling them by 0.89 . The experimental spectrum is taken from Ref. [36].

The experimental oscillator strengths do sum to approximately one, as expected. The sum of the theoretical oscillator strengths will be considerably higher than one, particularly if the transitions in the ultraviolet are included. Note, however, that oscillator strengths calculated by the ZINDO program are generally too large since the CI includes only singly excited configurations.

\section{Conclusions}

Geometry optimizations of the phthalocyanines $\mathrm{H}_{2} \mathrm{Pc}, \mathrm{CuPc}, \mathrm{SnPc}$, and $\mathrm{PbPc}$ have been carried out using ab initio calculations. DFT calculations were successful in obtaining the expected symmetries of $D_{2 \mathrm{~h}}$ for $\mathrm{H}_{2} \mathrm{Pc}, D_{4 \mathrm{~h}}$ for $\mathrm{CuPc}$, and $C_{4 \mathrm{v}}$ for $\mathrm{SnPc}$ for the equilibrium geometries, whereas the Hartree-Fock calculations yielded geometries slightly displaced from these symmetries, in having only $C_{2 \mathrm{v}}$ symmetry for $\mathrm{H}_{2} \mathrm{Pc}$ and $\mathrm{CuPc}$, and $C_{\mathrm{s}}$ symmetry for $\mathrm{SnPc}$ and $\mathrm{PbPc}$. The lower symmetry obtained in the HartreeFock calculations can be explained by the insufficient inclusion of electron correlation in the single determinant Hartree-Fock method. The calculated geometries are compared with experimental crystal structures, which deviate from the expected symmetries due to neighboring molecule effects in the crystal. In general, the agreement between the calculated and observed geometries is good, with the DFT results generally exhibiting a better agreement with experiment. The SCRF method for including solvent effects in the HF calculation had a small, nearly negligible, effect on the molecular structure of $\mathrm{SnPc}$, whereas the DFT/COSMO model yielded the expected result of increasing the $\mathrm{Sn}-\mathrm{N}$ bond length by pulling the $\mathrm{Sn}$ atom farther out of the molecular plane. The vibrational spectra calculated by the Hartree-Fock method are in only partial agreement with the experimental spectra. The electronic spectra calculated using the ZINDO program agree best with the measured vapor-phase spectra when the higher-symmetry LDA geometry is used. When the lower symmetry geometries are used, the characteristically strong B-band spectral line is split into several weaker lines.

\section{References}

[1] J.W. Perry, K. Mansour, I.-Y.S. Lee, X.-L. Wu, P.V. Bedworth, C.-T. Chen, D. Ng, S.R. Marder, P. Miles, T. Wada, M. Tian, H. Sasabe, Science 273 (1996) 1533.

[2] J.S. Shirk, R.G.S. Pong, F.J. Bartoli, A.W. Snow, Appl. Phys. Lett. 63 (1993) 18.

[3] J.W. Perry, K. Mansour, S.R. Marder, K.J. Perry, D. Alvarez Jr., I. Choong, Optics Lett. 19 (1994) 625.

[4] J.S. Shirk, R.G.S. Pong, S.R. Flom, M.E. Boyle, A.W. Snow, in: Materials for Optical Limiting, Materials Research Society Symposium Proceedings, vol. 374, 1995, p. 201.

[5] M.G. Cory, H. Hirose, M.C. Zerner, Inorg. Chem. 34 (1995) 2969.

[6] C.J. Brown, J. Chem. Soc. A: (1968) 2488.

[7] R. Kubiak, J. Janczak, J. Alloys Compd. 189 (1992) 107.

[8] K. Ukei, Acta Crystallogr. Sect. B: 29 (1973) 2290.

[9] Y. Iyechika, K. Yakushi, I. Ikemoto, H. Kuroda, Acta Crystallogr. Sect. B: 38 (1982) 766.

[10] B.F. Hoskins, S.A. Mason, Chem. Commun. (1969) 554.

[11] B. Szczepaniak, P. Bragiel, Vacuum 46 (1995) 465. 
[12] M.W. Schmidt, K.K. Baldridge, J.A. Boatz, S.T. Elbert, M.S. Gordon, J.J. Jensen, S. Koseki, N. Matsunaga, K.A. Nguyen, S. Su, T.L. Windus, M. Dupuis, J.A. Montgomery, J. Comput. Chem. 14 (1993) 1347.

[13] B. Delley, J. Chem. Phys. 92 (1990) 508.

[14] W.J. Hehre, R.F. Stewart, J.A. Pople, J. Chem. Phys. 51 (1969) 2657.

[15] W.J. Hehre, R. Ditchfield, R.F. Stewart, J.A. Pople, J. Chem. Phys. 52 (1970) 2769.

[16] J.S. Binkley, J.A. Pople, W.J. Hehre, J. Am. Chem. Soc. 102 (1980) 939.

[17] R. Ditchfield, W.J. Hehre, J.A. Pople, J. Chem. Phys. 54 (1971) 724.

[18] W.J. Hehre, R. Ditchfield, J.A. Pople, J. Chem. Phys. 56 (1972) 2257.

[19] M.M. Francl, W.J. Pietro, W.J. Hehre, J.S. Binkley, M.S. Gordon, D.J. DeFrees, J.A. Pople, J. Chem. Phys. 77 (1982) 3654.

[20] P.C. Hariharan, J.A. Pople, Theoret. Chim. Acta 28 (1973) 213.

[21] W.J. Stevens, H. Basch, M. Krauss, J. Chem. Phys. 81 (1984) 6026.

[22] W.J. Stevens, H. Basch, M. Krauss, P. Jasien, Can. J. Chem. 70 (1992) 612.
[23] T.R. Cundari, W.J. Stevens, J. Chem. Phys. 98 (1993) 5555.

[24] J.G. Kirkwood, J. Chem. Phys. 2 (1934) 351.

[25] L. Onsager, J. Am. Chem. Soc. 58 (1936) 1486.

[26] O. Tapia, O. Goscinski, Mol. Phys. 29 (1975) 1653.

[27] M.M. Karelson, A.R. Katritzky, M.C. Zerner, Int. J. Quantum Chem. Symp. 20 (1986) 521.

[28] K.V. Mikkelsen, H. Aagren, H.J.A. Jensen, T. Helgaker, J. Chem. Phys. 89 (1988) 3086.

[29] M.W. Wong, M.J. Frisch, K.B. Wiberg, J. Am. Chem. Soc. 113 (1991) 4776.

[30] M. Szafran, M. Karelson, A.R. Katritzky, J. Koput, M.C. Zerner, J. Comput. Chem. 14 (1993) 371.

[31] M. Karelson, T. Tamm, M.C. Zerner, J. Phys. Chem. 97 (1993) 11901.

[32] A. Klamt, G. Schuurmann, J. Chem. Soc. Perkin Trans. 2: (1993) 799.

[33] Biosym/MSI 3.0.0 release notes, October, 1995, pp. 23-34.

[34] J.E. Ridley, M.C. Zerner, Theoret. Chim. Acta 32 (1973) 111.

[35] J. Almlof, T.H. Fischer, P.G. Gassman, A. Ghosh, M. Haser, J. Phys. Chem. 97 (1993) 10964.

[36] T. Kobayashi, Spectrochim. Acta Part A: 26 (1970) 1313.

[37] M. Okawara, T. Kitao, T. Hirashima, M. Matsuoka, Organic Colorants, Kodansha Ltd., Tokyo, 1988, pp. 374-375.

[38] G.H. Heilmeier, G. Warfield, J. Chem. Phys. 38 (1963) 893. 